\title{
Fate of the right ventricle after fenestrated right ventricular exclusion for severe neonatal Ebstein anomaly
}

Brian L. Reemtsen, MD, Anastasios C. Polimenakos, MD, Brian T. Fagan, MD, Winfield J. Wells, MD, and Vaughn A. Starnes, MD

From Childrens Hospital Los Angeles and the Keck School of Medicine, Los Angeles, Calif.

Read at the Eighty-seventh Annual Meeting of The American Association for Thoracic Surgery, Washington, DC, May 5-9, 2007.

Received for publication May 2, 2007; revisions received July 10, 2007; accepted for publication July 16, 2007.

Address for reprints: Brian Reemtsen, MD, Childrens Hospital Los Angeles, Cardiothoracic Surgery, 4650 Sunset Blvd, Los Angeles, CA 90027. (E-mail: breemtsen@ chla.surgery.edu).

J Thorac Cardiovasc Surg 2007;134:1406-12 $0022-5223 / \$ 32.00$

Copyright $(9) 2007$ by The American Association for Thoracic Surgery

doi:10.1016/j.jtcvs.2007.07.047
Objectives: Neonates with Ebstein anomaly can have severe left ventricular dysfunction caused by right ventricular enlargement and ventricular septal displacement. Fenestrated right ventricular exclusion and Blalock-Taussig shunt (Starnes procedure) have been performed to mitigate this problem; however, the fate of the excluded ventricle and its effect on the systemic ventricle have yet to be documented.

Methods: Intermediate-term data have been retrospectively collected on the 12 survivors of neonatal right ventricular exclusion. Echocardiographic examinations were compared from the time of the original right ventricular exclusion and before second-stage Glenn palliation. Measurement of the Great Ormond Street ratio (area of right atrium plus atrialized right ventricle divided by area of trabeculated right ventricle plus left atrium and left ventricle), ratio of right ventricular to left ventricular area, degree of ventricular septal impingement into the left ventricle, and left ventricular shortening fraction have been documented.

Results: In the 12 survivors of right ventricular exclusion, a decrease in the mean Great Ormond Street ratio was observed (before fenestrated right ventricular exclusion: $1.04 \pm 0.49$ vs before Glenn palliation: $0.31 \pm 0.10, P=.01$ ). The average pre-Glenn right ventricular/left ventricular ratio was substantially less than 1.0 (mean, $0.63 \pm 0.27$ ), demonstrating right ventricular regression. The degree of left ventricular septal impingement decreased by an average of $38 \%(P=.008)$, normalizing left ventricular morphology. At the time of Glenn palliation, the left ventricular shortening fraction was normal in all patients (mean, $42 \% \pm 7 \%$ ).

Conclusions: After neonatal right ventricular exclusion (Starnes procedure) for severe Ebstein anomaly, there is a reduction in right ventricular size, as demonstrated by echocardiographic evidence of a significant decrease in Great Ormond Street ratios. This regression correlates with ventricular septal realignment and normalization of left ventricular function.

$\mathrm{N}$ eonates presenting with a combination of severe Ebsteinoid changes of the tricuspid valve and variable amount of right ventricular outflow tract (RVOT) obstruction continue to represent a clinical conundrum with low survival. ${ }^{1,2}$ Our group has recently published the details of a series of patients who underwent a univentricular repair consisting of fenestrated right ventricular (RV) exclusion and modified Blalock-Taussig (BT) shunt (Starnes procedure) with acceptable survival. ${ }^{3}$ Our objective in this study was to characterize the effect of fenestrated RV exclusion on both the right ventricle and the systemic ventricle.

We have used standard tomographic and echocardiographic measurements in an attempt to describe the proportional regression of the excluded ventricle and the normalization of the morphology and function of the systemic ventricle. 


$$
\begin{aligned}
& \text { Abbreviations and Acronyms } \\
& \begin{aligned}
\mathrm{BT} & =\text { Blalock-Taussig } \\
\mathrm{CT} \text { ratio } & =\text { preoperative ratio of the cardiac silhouette } \\
& \text { to the thoracic cage } \\
\mathrm{GOS} & =\text { Great Ormond Street } \\
\mathrm{LV} & =\text { left ventricular } \\
\mathrm{RV} & =\text { right ventricular } \\
\mathrm{RVOT} & =\text { right ventricular outflow tract }
\end{aligned}
\end{aligned}
$$

\section{Materials and Methods}

A retrospective review of intermediate-term data (median followup, 30 months; range, 6-204 months) for 12 of 16 survivors with critical Ebstein anomaly who underwent RV exclusion as neonates was performed. Echocardiographic examinations were compared at the time of the original RV exclusion, before second-stage Glenn palliation, and before the Fontan procedure to determine the fate of the excluded ventricle and its effect on the systemic ventricle. Institutional approval was obtained before the inception of the study.

\section{Demographics and Preoperative Data}

Of the 12 survivors, 5 were girls and 7 were boys, with a mean weight of $3.05 \pm 0.45 \mathrm{~kg}$. One was premature (36 weeks' gestation). Seven had a prenatal diagnosis made on the basis of fetal echocardiography. All the survivors had a patent ductus arteriosus and atrial septal defect at the time of the diagnosis. Seven patients had severe pulmonary valve stenosis or atresia. All 12 patients underwent pre-Glenn and 7 underwent pre-Fontan assessment, including echocardiographic analysis and catheterization. All patients showed favorable hemodynamics and were deemed a suitable risk for bidirectional Glenn shunt or progression to completion of the Fontan procedure.

\section{Tomographic Assessment}

Standard anterior-posterior tomographic analysis was performed preoperatively, at the time of Glenn palliation, and at the time of final Fontan palliation. The cardiac silhouette was measured and compared with the length of the thoracic cage at the same level. This number determines the preoperative ratio of the cardiac silhouette to the thoracic cage (CT ratio).

\section{Echocardiographic Assessment}

The echocardiographic assessment was performed preoperatively before the original RV exclusion, the Glenn procedure, and Fontan completion respectively. Four separate variables were measured for evaluation of the excluded right ventricle and systemic ventricle at both time points.

First, a Great Ormond Street (GOS) ratio was calculated, as described by Celermajer and associates. ${ }^{4}$ The GOS ratio is determined as a product of the combined area of the right atrium and atrialized portion of the right ventricle divided by the area of the trabeculated right ventricle plus the left atrium and ventricle. As an arbitrary expression of the GOS ratio, the score represents a graded severity correlate of the mortality risk. Grade 1 is characterized as a ratio of less than 0.5 , grade 2 as a ratio of 0.5 to 0.99 , grade 3 as

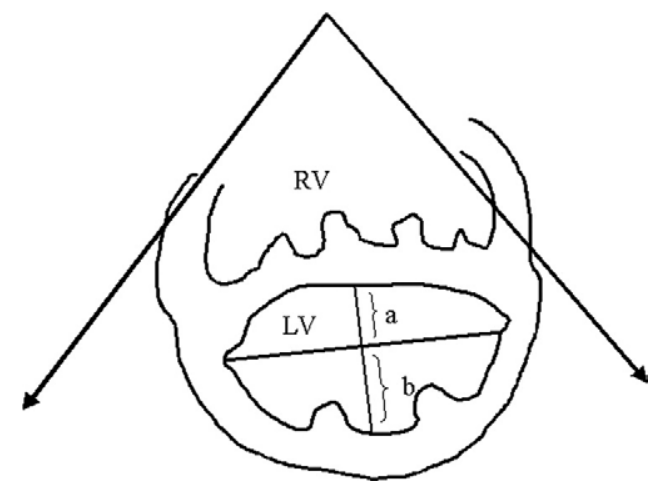

Figure 1. Schematic illustration of the parasternal short-axis view. The septal impingement ratio is calculated at the endsystolic phase and equals $a / b$ values measured in the left ventricle ( $L V$ ). Values approaching $\mathbf{1 . 0}$ indicate circular morphology, and values of less than 1.0 connote septal impingement. $R V$, Right ventricle.

a ratio of 1.0 to 1.49 , and grade 4 as a ratio of equal to or greater than 1.5. A GOS score of grade 3 has been associated with a substantially higher mortality. ${ }^{4}$

Second, to measure the degree of the effective remodeling of the trabeculated RV area in relation to the functional left ventricle, the RV/left ventricular (LV) ratio was calculated. The RV and LV areas were measured in an apical 4-chamber echocardiographic view. This ratio correlates with the degree of the RV regression during the time interval. Because of the "redefinition of the RV" at initial palliation, the RV/LV ratio was calculated before Glenn palliation and before the Fontan procedure only.

Third, the degree of septal remodeling in relation to the LV morphology was assessed by using the LV septal impingement ratio. This ratio is the product of the upper septal curvature vertical dimension from the RV wall (length connoted by $a$ in Figure 1) divided by the lower septal curvature vertical dimension from the LV wall (length connoted by $b$ in Figure 1), as measured in the short-axis, left parasternal echocardiographic view. This ratio indirectly correlates with the degree of LV morphology normalization as a result of the LV septal impingement regression (Figure 1). ${ }^{5}$

Last, the LV shortening fraction was calculated and compared by using standard echocardiographic techniques. ${ }^{6}$

\section{Surgical Technique}

A univentricular (fenestrated RV exclusion) strategy was undertaken as the original first-stage palliative approach for all the patients who eventually qualified for the bidirectional Glenn procedure. All 12 survivors had a tricuspid valve not amenable to repair with an inadequate functional portion of the right ventricle associated with variable amounts of RVOT obstruction not amenable to a hemodynamically acceptable reconstruction.

Standard aortic and bicaval cannulation are used. During moderate hypothermia, the heart is arrested with cold blood cardioplegia. The tricuspid valve is approached through an oblique right atriotomy incision. The RV fenestrated exclusion was accomplished by patching the tricuspid valve at the "anatomic" level of 
TABLE 1. Tomographic and echocardiographic measurements

\begin{tabular}{lccc}
\hline & $\begin{array}{c}\text { Preoperative } \\
(\mathbf{n}=\mathbf{1 2})\end{array}$ & $\begin{array}{c}\text { Pre-Glenn } \\
(\mathbf{n}=\mathbf{1 1})\end{array}$ & $\begin{array}{c}\text { Pre-Fontan } \\
(\mathbf{n}=\mathbf{6})\end{array}$ \\
\hline CT ratio & $0.84 \pm 0.09$ & $0.56 \pm 0.1^{*}$ & $0.52 \pm 0.08^{*}$ \\
GOS ratio & $1.04 \pm 0.49$ & $0.31 \pm 0.01^{*}$ & $0.26 \pm 0.05^{*}$ \\
RV/LV ratio & & $0.63 \pm 0.27$ & $0.44 \pm 0.05^{*}$ \\
Septal impingement & $0.58 \pm 0.09$ & $0.93 \pm 0.13^{*}$ & $1.01 \pm 0.17^{*}$ \\
Shortening fraction $(\%)$ & $31 \% \pm 4 \%$ & $42 \% \pm 7 \%$ & $43 \% \pm 4 \%$
\end{tabular}

CT ratio, Preoperative ratio of the cardiac silhouette to the thoracic cage; $G O S$, Great Ormond Street; $R V$, right ventricular; $L V$, left ventricular. ${ }^{*} P \leq$ .05 .

its annulus by using glutaraldehyde-fixed autologous pericardium. The coronary sinus is not incorporated under the pericardial exclusion and therefore is left in the anatomic position in the morphologically right atrium. A 4- to 5-mm fenestration is performed on the patch by using a coronary punch. Free atrial communication is ensured, and a right atrial reduction plasty is carried out as a standard component of the fenestrated RV exclusion strategy. If pulmonary artery insufficiency exists, pulmonary artery interruption is performed. Early RV exclusion did incorporate RV plication, if necessary, but we have abandoned this practice in the current era because of RV involution observed after palliation. Finally, pulmonary blood flow is provided by a modified BT shunt $(3.5-4.0 \mathrm{~mm})$.

\section{Results}

From 1992 to the present, 16 patients with severe Ebstein anomaly have undergone RV exclusion with a modified BT shunt at a single institution, with 12 survivors. The first survivor had an unfenestrated tricuspid patch, and all other survivors had the fenestration. Eleven of 12 survivors have gone on to a bidirectional Glenn shunt, and 6 patients have been completely palliated with total caval pulmonary connection. There have been no interstage deaths. No hospital mortality has been observed in this treatment group since 1996. To this point, all patients have been evaluated and deemed acceptable candidates for bidirectional Glenn and Fontan procedures at the age-appropriate time. One patient has a pacemaker for complete heart block after RV exclusion and underwent a modified maze procedure for severe preoperative tachyarrythmias.

\section{CT Ratio}

The CT ratio was observed to be $0.84 \pm 0.09$. At the time of the Glenn shunt, the ratio had decreased to $0.56 \pm 0.10$; this difference was statistically significant to a $P$ value of .003. The CT ratio did not significantly change in the time between the Glenn and Fontan operations (Table 1).

\section{GOS Ratio}

In the 12 survivors of RV exclusion, a decrease in the mean GOS ratio was observed (before fenestrated RV exclusion:
$1.04 \pm 0.49$ vs before Glenn palliation: $0.31 \pm 0.10, P=$ $.01)$. There was a uniform decrease in all survivors. No difference could be elucidated among the fenestrated or nonfenestrated groups because of the low numbers of patients. The pre-Fontan values observed demonstrated a continued significant decrease from preoperative ratios, with a mean of $0.26 \pm 0.05$. This value was statistically significantly lower than preoperative measurements. Overall, the ratio trend was less than at Glenn palliation, but the value did not reach statistical significance. The GOS score equals 1 at Glenn palliation and Fontan completion in all patients.

\section{RV/LV Ratio}

The $\mathrm{RV} / \mathrm{LV}$ ratio was measured as $0.63 \pm 0.27$ at the time of the Glenn shunt. This value continued to decrease to a mean of $0.44 \pm 0.05$ measured at the time of the Fontan procedure. Both of these values are significantly less than the expected 1:1 ratio expected with normal anatomy. This value represents the continued comparative regression of the right ventricle from the left ventricle during the interim between the Glenn and Fontan procedures.

\section{Septal Impingement}

As shown in Figure 1, $A$, a calculated a/b ratio approaching 1.0 connotes a circular structure, whereas a value approaching zero connotes a flattened structure. This ratio helps to identify the degree of septal impingement and its effect on the shape and presumed function of the left ventricle. As shown in Figure 2, preoperative septal impingement was calculated preoperatively at a mean of $0.58 \pm 0.09$, whereas the values increased significantly to $0.93 \pm 0.13$ and $1.01 \pm$ 0.17 , respectively, at the Glenn and Fontan procedures $(P=$ .008 , Figure 3 ). This difference clearly establishes the RV dilation's effect on the LV morphology seen in severe neonatal Ebstein anomaly, as well as documenting the normalization of the left ventricle after palliation.

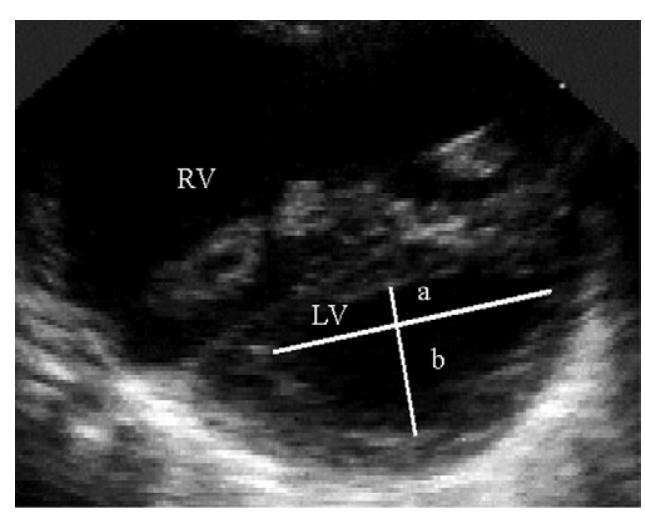

Figure 2. Two-dimensional echocardiogram of the parasternal short axis. This is a preoperative view demonstrating septal impingement, with a/b approaching zero. $R V$, Right ventricle; $L V$, left ventricle. 


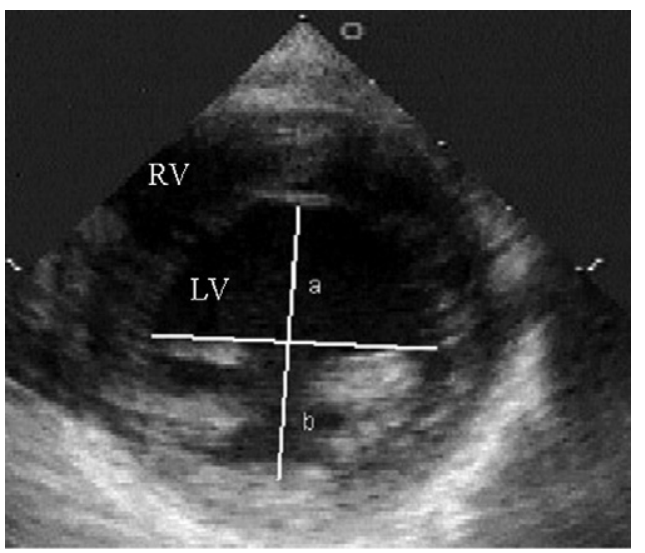

Figure 3. Two-dimensional echocardiogram of the parasternal short axis. This is a pre-Glenn view demonstrating lack of septal impingement, with a/b approaching 1.0, suggesting normal morphology of the left ventricle (LV. RV, Right ventricle.

\section{Shortening Fraction}

The patients' left ventricles were in general severely affected, with a mean preoperative shortening fraction of $31 \% \pm 4 \%$ with inotropic support. These values significantly improved $(42 \% \pm 7 \%$ at Glenn palliation and $43 \% \pm$ 4 at the Fontan procedure) when measured at the respective preoperative visits.

We observed a decrease in the relative and net size of the right ventricle after exclusion. With this measurable regression, we have documented normalization of both morphology and function of the left ventricle, leading to effective palliation and increased survival.

\section{Discussion}

Although the fenestrated RV exclusion approach to palliation for severe Ebstein anomaly has demonstrated improved survival, no documentation of the physiologic effect has been published. ${ }^{7}$ Our study has implied 2 major ideas. First, children with severe Ebstein anomaly have not only severe right-sided failure but also biventricular failure. The dilation of the right side of the heart causes septal impingement and resultant left-sided heart failure. Second, the fenestrated RV exclusion induces a durable regression of the right-sided structures, thereby restoring the systemic ventricular morphology and function. The postoperative reestablishment of normal ventricular function clearly demonstrates the ventricular interplay that leads to severe heart failure in these neonates.

Although others contend that all patients with severe Ebstein anomaly can have this condition repaired, we continue to believe that patients with poor valve delamination and the combination of a diminutive trabeculated right ventricle with RVOT obstruction are better served with a single-ventricle repair. ${ }^{8}$ At the severe end of the spectrum, maximal decompression and subsequent involution of the right-sided structures after RV exclusion should not only allow patients to survive the neonatal period but also make them excellent candidates for future total cavopulmonary connection.

Other derivations of the RV exclusion have favored wide plication and even excision of the atrialized portion of the right ventricle seen in severe Ebstein anomaly. ${ }^{9,10}$ The involution of the right-sided structures seen after fenestrated RV exclusion obviates the need for RV manipulation. This process should serve to decrease operative times and spare any possible injury to the right coronary artery made possible with RV excision or excessive plication. ${ }^{11}$ Other processes, apart from severe Ebstein anomaly, causing RV failure cannot be commented on based on our current study.

No study to date has described RV function or its effect on the systemic ventricle after valve repair for severe neonatal Ebstein anomaly. Chauvaud and colleagues ${ }^{12}$ have described increased LV ejection fraction correlating with decreased end-diastolic RV volume after plication and valve repair in adult patients; however, long-term follow-up has not been included. The majority of valve repair articles describe postoperative patients as asymptomatic in New York Heart Association Class I, without physiologic parameters of ventricular function. To date, we have seen uniform $\mathrm{RV}$ regression in our patients and normalization of the $\mathrm{LV}$ function, which is durable. In the short term, this leads to better systemic ventricular mechanics in these critically ill children and improves survival. We have encountered and expect excellent Fontan candidates because of this normalization of the LV morphology and function.

\section{References}

1. Yetman AT, Freedom RM, McCrindle BW. Outcome in cyanotic neonates with Ebstein's anomaly. Am J Cardiol. 1998;81:749-54.

2. Celermajer DS, Bull C, Till JA, et al. Ebstein's anomaly: presentation and outcome from fetus to adult. J Am Coll Cardiol. 1994;23:170-6.

3. Reemtsen BL, Fagan BT, Wells WJ, Starnes VA. Current surgical therapy for Ebstein anomaly in neonates. $J$ Thorac Cardiovasc Surg. 2006;132:1285-90.

4. Celermajer DS, Cullen S, Sullivan ID, Spiegelhalter DJ, Wyse RK, Deanfield JE. Outcome in neonates with Ebstein's anomaly. J Am Coll Cardiol. 1992;19:1041-6.

5. Satomi G, Nakamura K, Takao A, Imai Y. Two-dimensional echocardiographic detection of pulmonary venous channel stenosis after Senning operation. Circulation. 1983;68:545-9.

6. Shors CM. Cardiac function determined by echocardiogram. Crit Care Med. 1975;3:5-7.

7. Knott-Craig CJ, Overholt ED, Ward KE, Razook JD. Neonatal repair of Ebstein's anomaly: indications, surgical technique, and mediumterm follow-up. Ann Thorac Surg. 2000;69:1505-10.

8. Knott-Craig CJ, Overholt ED, Ward KE, Ringewald JM, Baker SS, Razook JD. Repair of Ebstein's anomaly in the symptomatic neonate: an evolution of technique with 7-year follow-up. Ann Thorac Surg. 2002;73:1786-93. 
9. Yun IT, Lee SH, Ko JK. Neonatal stenotic Ebstein's anomaly: a novel technique of right ventricular exclusion. J Thorac Cardiovasc Surg. 2005;131:469-71.

10. Sano S, Ishino K, Kawada M, et al. Total right ventricular exclusion procedure: an operation for isolated congestive right ventricular failure. J Thorac Cardiovasc Surg. 2002;123:640-7.

11. Chauvaud S. Ebstein's malformation. Surgical treatment and results. J Thorac Cardiovasc Surg. 2000;48:220-3.

12. Chauvaud SM, Hernigou AC, Mousseaux ER, Sidi D, Hebert JL. Ventricular volumes in Ebstein's anomaly: X-ray multislice computed tomography before and after repair. Ann Thorac Surg. 2006;81: 1443-9.

\section{Discussion}

Dr V. Mohan Reddy. (Stanford, Calif). I would like to thank the association for the privilege of discussing this manuscript. I would also like to thank the authors for providing me with the manuscript early enough and congratulate them for an elegant presentation and study.

Dr Reemtsen and colleagues report on a 15-year single-institution experience of neonatal Ebstein's anomaly with an overall mortality of $25 \%$ but none since 1996 . This approach commits all patients to single - ventricle circulation. Probably some of these could have been a 2 -ventricle or a $1 \frac{1}{2}$-ventricle type of repair based on our experience and review of the literature on neonatal Ebstein's anomaly. It might be that although this approach yields very good survival, a more balanced individualized approach might yield similar results without committing all patients to single-ventricle repairs.

In your manuscript, at least, I would say that $50 \%$ of the patients have a GOS ratio of less than 1 . Perhaps in these patients a 2-ventricle or a 1/2-ventricle type of repair could be attempted as a first option.

Also, by means of right atrial reduction and RV plication, with or without, the GOS ratio immediately decreases if you do a postoperative study. But what is more important in your manuscript is the long-term follow-up and continued remodeling of the ventricle and the septum. This is, I think, the strength of your study.

The authors state that these patients were all catheterized but do not present any data on the catheterization. I have 4 or 5 questions for you, and I would appreciate if you could answer each one of them before I move on to the next.

Do you currently adopt this approach in all neonates or consider 2-ventricle repair in some? If so, what criteria do you use?

Dr Reemtsen. Our goal, off the bat, is to establish a 2-ventricle physiology, if possible, but I think that if you look at some of the patients in our group, they have the massively dilated atrialized portion of the right ventricle and really very little trabeculated right ventricle. More to the point, the more significant thing is that they really have a lack of functional tricuspid valve tissue altogether, and therefore it is possible to do a repair, but I do not think you would be doing the patient any favors.

Dr Reddy. So in the last 15 years, you have not attempted a 2-ventricle repair?

Dr Reemtsen. Actually, we have, and generally, these 2ventricle repairs are not in the neonatal time frame. We have had 3 total repairs during that time. We have had 1 death, 1 conversion to the Starnes procedure, and 1 that was successful. The last patient had the kind of tripartite ventricle that we would expect to perform well.
Another and equally significant lesion we worry about is pulmonary stenosis or atresia, which is commonly seen in neonates with severe Ebstein's anomaly. Because if you are doing a 2ventricle repair, again we are saying that you have leaflet tissue that is not perfect, and then you also have RVOT obstruction. You are going to put a small conduit in a neonate, and we know the implications of that. Therefore in those patients especially, if they have a ventricle that is kind of on the line and they have associated pulmonary stenosis or atresia, we will go to the single-ventricle approach.

Dr Reddy. You have catheterized most of the patients before the Glenn and Fontan procedures. Do you have any information on the end-diastolic pressure on these ventricles and the cardiac index?

Dr Reemtsen. I have no specific numbers, but I actually looked through this before I left. All of these patients who we have observed thus far have been very good Fontan candidates, and we expect that. Most of that is in part due to the normalization that we see with the left ventricle.

Dr Reddy. I notice you do fenestrated patches in most of these, and also from your manuscript I saw that when there is severe pulmonary stenosis, you chose to entrap the pulmonary artery. But in patients with antegrade flow, do you close this fenestration when you do a Glenn or a Fontan procedure?

Dr Reemtsen. No, we leave the fenestration open. Really, we only will interrupt the pulmonary artery if there is significant regurgitation because we just want to keep the right ventricle decompressed. That is the main goal. Therefore if there is antegrade flow and no regurgitation, we would leave that alone, but that does not happen very often. As I said, the majority of these patients have severe pulmonary stenosis or functional pulmonary atresia.

Dr Reddy. The reason I ask that is that if you have antegrade flow, you could close the fenestration. If you leave the fenestration open, it is sort of acting like tracks for regurgitation or some sort of regurgitation. Obviously you have regurgitation, and if you have a single-ventricle type of physiology, any amount of regurgitation probably is not a good idea in the long run anyway.

Dr Reemtsen. Well, the main point is that these are almost nonfunctional right ventricles, and essentially, we just leave the fenestration there in case there is any flow from the thebesian veins because these are not normal ventricles. It is really a pop-off, and the natural history is regression of this ventricle. Therefore we just want to make sure that there is complete decompression, and that is why we keep the fenestration patent.

Dr Reddy. That leads to my last question. You said that most of these ventricles are nonfunctional and very small. Have you noticed any thromboembolic phenomenon from this stasis?

Dr Reemtsen. We have not. We have not documented anything like that.

Dr Reddy. Again, congratulations.

Dr Reemtsen. Thank you.

Dr Shunji Sano. (Okayama City, Japan). I have some comments and questions. Septal impingement (SI ratio $=a / b)$ in echocardiographic analysis is actually the ratio to measure the $\mathrm{RV} / \mathrm{LV}$ pressure ratio, although this will become abnormal at the time of volume overload. Therefore this does not help to measure the volume load ratio in patients with Ebstein's anomaly. 
Recovery of LV function, recovering after the RV exclusion, is mostly based on the normalization of the intraventricular septum. The LV end-diastolic and end-systolic dimensions that we can measure became enlarged soon after the RV exclusion because of the changes of ventricular septal motion. This phenomenon is seen in patients with atrial septal defects with a huge Qp/Qp ratio. These are all due to geometric changes.

Another thing is that in your slides the CT ratios and also the echocardiograms show the improvement of ventricular LV motion, and also the $\mathrm{CT}$ ratio decreased before the Glenn procedure. From my experience of RV exclusion operations, when you excise the LV free wall to decrease RV volume, intraventricular septal motion changed to normal from a paradoxical value soon after the RV exclusion, and the CT ratio also decreased soon after. Therefore another question is this: in the Starnes operation, without a complete right LV exclusion, when do these findings, such as IVS motion and CT ratio change, occur, soon after the operation or a few months later?

Dr Reemtsen. No, I think it is certainly not immediate. Essentially, after we perform this procedure, sometimes they have pulmonary vascular twitchiness, and I think that is in response to their LV failure. Usually we have to keep them on nitric oxide for a couple of days to try and ameliorate that. Therefore I think it is more than a week's time.

However, you can notice, as Dr Reddy said, that as soon as you do this resection, the GOS ratio should decrease. But looking at $\mathrm{X}$-ray films immediately postoperatively, it is not as marked, and it is not even close to as marked as we see at the time of the Glenn procedure. Therefore I think there is an immediate improvement over a week-long postoperative period, and I think we have clearly shown that there is continued improvement or regression and normalization of the function of the left ventricle.

Dr Sano. I must inform you that from our experience, the RV size decreased because our aim was to decrease RV size to excise the RV free wall, but before the Glenn procedure, the right ventricle increased again. Therefore it is almost similar to your echocardiographic findings. Therefore we excise the RV free wall again in few patients when the right ventricle increased again. I do not know whether it is due to direct anastomosis of the RV free wall after we excised the RV free wall or not. I wonder if I excise the $\mathrm{RV}$ free wall and put a small polytetrafluoroethylene patch, whether the right ventricle will increase in size or not. I do not know.

Dr Reemtsen. I should not ask you a question, but did you have any pulmonary atresia? Did you have a conduit in place or anything like that that would kind of be the substrate for this kind of redilation of your right ventricle?

Dr Sano. Yes, we have patients with true pulmonary atresia. No, we have not used a conduit.

Dr Reemtsen. That would be an issue.

Dr Joseph A. Dearani. (Rochester, Minn). I would just like to make one comment in follow-up to Dr Reddy's comment about this dilemma and trying to decide on a single-ventricle pathway or biventricular repair in the neonatal period. One piece of information that I will share with you that will be presented in our Ebstein's anomaly presentation tomorrow is that anatomic obstruction at the RV outflow level or the pulmonary valve level is an independent predictor for mortality in late reoperation in a very large number of patients with Ebstein's anomaly. Consequently, if an anatomic obstructive lesion in the RVOT or the pulmonary valve level is demonstrated in the neonate, then we believe the single-ventricle pathway is preferred.

Dr Reemtsen. And I can tell you from our experience that we had 3 patients in this series, not of the RV exclusion, but all of the neonates who underwent intervention, and every patient who had a homograft placement as a neonate was either an early or late death. Therefore we observed $100 \%$ mortality with placement of conduits in the neonates.

Dr William J. Brawn. (Birmingham, United Kingdom). Is it the shunt that saves the neonate's life? Is that the essential part of the operation?

Dr Reemtsen. No, it is not. We have a great example of a patient who came with this problem last year, and we just did a shunt first. The patient struggled along with persistent need for not only inotropes but also ventilatory support for 2 weeks. We went in and revised him to the Starnes procedure, and the kid was off the vent within a few days. Therefore I think it is not only the pulmonary blood flow issue, it is this interventricular relationship and this dilation that you need to ameliorate.

Dr Glen Van Arsdell. (Toronto, Ontario, Canada). I hesitate to even discuss this because the results are so good, but one of the things this does is it forces you to make a decision early on as to whether it is a 2-ventricle or single-ventricle repair. We have taken an alternative approach of saying the primary issue is that there is high PVR in the ductus in many of these kids staying open and let us ligate the duct, let us alkalinize them and start them on nitric oxide. Obviously that only works when it is functional pulmonary atresia. Therefore I wonder whether you have explored that possibility. These patients who are labeled as having atresia, I know you have tried to classify them. Have you proved actual atresia, or is it echocardiographic atresia that only looks that way because you have high pulmonary artery pressures related to a duct that is open?

Dr Reemtsen. No, it is significant pulmonary stenosis, and almost all of them have pulmonary stenosis of some sort. However, the amount of pulmonary atresia was more than half the patients that we said had actual pulmonary atresia.

Dr Van Arsdell. Therefore for those patients, this is clearly a very good strategy.

The question, I guess, is this: For the other patients, can you go with a high PVR route and buy some time, maybe you buy them a year or 2 or 3 before you have to make the next decision?

Dr Reemtsen. We would certainly attempt that first. There is no question that we would-we always attempt to treat these patients medically first. It is not a kind of whisk off to the operating room once we have this diagnosis, but our goal would really be to get these patients out and not operate on them.

Dr Van Arsdell. But I mean specifically including duct ligation as part of a strategy and alkalinization and nitric oxide?

Dr Reemtsen. Certainly.

Dr George R. Daicoff. (St Petersburg, Fla). A number of years ago, I had a neonate such as you have presented, and when I reviewed the literature, there was a $100 \%$ mortality rate in this group. The heart was so huge. The lungs were hypoplastic and underdeveloped. The baby was on a ventilator. A review of the literature was not helpful with this desperate neonate, which led me to confer with Gordon Danielson, who had a large series of 
patients with Epstein's anomaly. He said that they did not have any neonates in their Mayo Clinic practice.

Therefore I took the baby to the operating room prepared to do an intracardiac procedure. The right atrium and right ventricle were both huge, filling the thorax. The tricuspid regurgitation was massive. In evaluating the tricuspid before cannulating for bypass, I inverted the free wall of the right atrium through the tricuspid annulus into the right ventricle. The tricuspid regurgitation became a lot less, if not eliminated completely. Furthermore, this maneuver decompressed and diminished the size of both right-sided chambers. It occurred to me that I could reproduce this situation with one stitch. Therefore I took a double-armed, pledgeted, plicating stitch through the anterior wall of the right ventricle; dodged my finger; and brought needles through the free wall of the right atrium and a second pledget. Tying the stitch brought the right atrium into the right ventricle. A modified BT shunt was done at the same time. He did amazingly well and subsequently underwent the Fontan operation. No cutting, no sewing; it was an easy operation. I could not believe it.
Therefore the question I have for you is this: If I could do this in the way I did, and probably obliterate the chamber, why do you need a fenestration at all?

Dr Reemtsen. Well, I think the fenestration really comes into account when you have what we are talking about: functional or true pulmonary atresia because the ventricle needs some outflow. I do not know about the patient that you had, if he or she had pulmonary atresia, and it sounds to me like he or she did not. If there is antegrade flow, the fenestration specifically might not be necessary.

Dr Daicoff. That BT shunt was for that purpose, right?

Dr Reemtsen. What purpose is that? I am sorry?

Dr Daicoff. To give the pulmonary blood flow.

Dr Reemtsen. Yes, I agree. What I am saying though is that if you have antegrade flow from out of the pulmonary valve, it will decompress this ventricle and you will not need the fenestration. If you have pulmonary atresia and you essentially occlude the right ventricle with thebesian flow, it is likely that this is going to dilate. Therefore that is the issue. 